\title{
Diseño metodológico de la encuesta sobre condiciones de seguridad y salud en el trabajo en Ecuador
}

\section{Methodological design of the survey on occupational health and safety conditions in Ecuador}

Silva Peñaherrera, M.G

Gómez García, A.R.

Vilaret Serpa, A.

Suasnavas Bermúdez, P.R.

Universidad Internacional SEK Ecuador, Ecuador

Autor para correspondencia: antonio.gomez@uisek.edu.ec

Fecha de recepción: 13 de Julio de 2016 - Fecha de aceptación: 30 de Agosto de 2016

Resumen: Introducción: Las encuestas sobre condiciones de trabajo y salud en el trabajo han demostrado ser un instrumento fiable para la recolección de información sobre factores de riesgo laboral; estas se han llevado a cabo, a nivel internacional y latinoamericano por reconocidos organismos, recolectando datos de relevante importancia tanto para el establecimiento de un diagnóstico situacional del país en seguridad y salud ocupacional, como para la formulación de políticas públicas. En Ecuador, hasta el presente año no se ha realizado una encuesta centrada a esta temática. Objetivo: Diseñar la Primera Encuesta sobre Condiciones de Seguridad y Salud en el Trabajo para Ecuador (I ECSST-E), que sirva de modelo de recogida de información para conocer los factores de riesgo en el trabajo, su impacto en la salud de la población trabajadora ecuatoriana y que permita la comparación de los resultados con los de encuestas internacionales. Metodología: La encuesta se diseñó por investigadores de la Universidad Internacional SEK, en 3 fases secuenciales que requirieron la participación de 40 investigadores y expertos en el área, en colaboración con instituciones universitarias y profesionales del sector privado, el diseño se estructuró buscando ajustarse al contexto del país, así como, a los estándares de encuestas internacionales y latinoamericanas, para lo que se realizó una exhaustiva revisión bibliográfica y consulta documental y estructural de trabajos relacionados y encuestas. Resultados: La encuesta tuvo como resultado 79 variables (preguntas) relacionadas con factores de riesgo ocupacional y salud percibida por el trabajador, estás se derivaron del consenso que el grupo de investigadores y expertos llegaron a través de las tres fases de diseño. Se determinaron 7 dimensiones (sociodemográfica y laboral, condiciones de empleo, seguridad en el trabajo, higiene industrial, ergonomía, psicosocial y salud ocupacional), 26 sub-dimensiones que derivaron en 79 preguntas que conformaron la Encuesta. Conclusiones: En este sentido, el presente modelo de encuesta sobre condiciones de seguridad y salud ocupacional pretende contribuir, modestamente, a otros estudios y encuestas realizadas en Latinoamérica y, sus resultados permitirán la comparación a nivel internacional.

Palabras clave: encuesta; instrumento; condiciones de trabajo; salud ocupacional; ecuador Abstract: Introduction: Surveys on working conditions and health at work have proven to be reliable for gathering information about occupational risk factors instrument; these have been 
carried out at the international and Latin American level by recognized bodies, collecting data relevant importance for the establishment of a country situational analysis in occupational safety and health, and for public policy. In Ecuador, until this year it has not conducted a survey focused on this subject. Objective: Design the First Survey on Conditions of Safety and Health at Work for Ecuador (I ECSST-E), which serve as a model for gathering information to know the risk factors at work, their impact on population health Ecuadorian worker and to permit comparison of results with those of international surveys. Methodology: The survey by researchers at the Universidad Internacional SEK was designed, in 3 sequential phases that required the participation of 40 researchers and experts in the field, in collaboration with university and private sector professionals institutions, the design was structured looking fit the context the country as well as international standards and Latin American surveys, for which a comprehensive literature review and documentary and structural consultation and surveys related work was performed. Results: The survey resulted in 79 variables (questions) related to perceived health worker and occupational risk factors, you were derived from the consensus that the group of researchers and experts came through the three phases of design. 7 dimensions (socio-demographic and employment, conditions of employment, occupational safety, industrial hygiene, ergonomics, psychosocial and occupational health), 26 sub-dimensions that led to 79 questions that formed the survey were determined. Conclusions: In this sense, the present model survey on safety and occupational health aims to contribute modestly to other studies and surveys conducted in Latin America and its results will allow comparison internationally.

Key words: survey instrument; working conditions; occupational health; Ecuador

\section{Introducción}

En los últimos años, a nivel internacional se han realizado encuestas sobre las condiciones de trabajo y salud laboral. En lo que se refiere a Latinoamérica se han ejecutado encuestas en Guatemala (2007) ${ }^{1}$, República de Colombia (2007 y 2013) ${ }^{2-3}$, Argentina (2009) ${ }^{4}$, Chile (2011) ${ }^{5}$, Uruguay $(2012)^{6}$ y Centroamérica $(2011)^{7}$. Un referente importante, son las encuestas a nivel nacional realizadas en España desde 1987, siendo la última la VII Encuesta Nacional de Condiciones de Trabajo de $2011^{8-9}$, así mismo, varias Comunidades Autónomas han llevado a cabo su propia encuesta ${ }^{10 ; 12}$.

En este campo de estudio, se evidencia un consenso mundial sobre la necesidad de conocer las condiciones de empleo, trabajo y estado de salud del trabajador ${ }^{13-14}$. No obstante, existen importantes diferencias en el diseño y metodología de las encuestas antes mencionadas ${ }^{15 ; 18 .}$

En el caso de Ecuador no existe una encuesta orientada en este ámbito, a excepción de la Encuesta de Condiciones de Vida $(E C V)^{19}$ orientada a estudiar los impactos económicos y las condiciones de vida de la población ecuatoriana y la Encuesta Nacional de Salud y Nutrición $\left(\right.$ ENSANUT) ${ }^{20}$.

Las encuestas sobre las condiciones de trabajo y salud permiten obtener información de los factores de riesgo laboral en sus diferentes dimensiones (seguridad, higiene, ergonomía, y psicosociología). La aplicación de un modelo correcto de recogida de información, valida los datos obtenidos, permite la comparabilidad entre países y ofrece un diagnóstico situacional de las condiciones de trabajo y cómo estos afectan al estado de salud de los trabajadores. 
Los resultados obtenidos a través de estas encuestas son de relevante importancia para la elaboración de políticas públicas, la evaluación de normativas vigentes, la creación y evaluación de programas preventivos que mejoren la salud de los trabajadores, así como, para establecer líneas prioritarias de investigación en materia de seguridad y salud ocupacional en el país.

Dada la importancia de esta información y siendo escasos los datos generados en Ecuador que evidencien el estado actual de las condiciones de seguridad y salud de los trabajadores, la Facultad de Ciencias del Trabajo y Comportamiento Humano de la Universidad Internacional SEK-Ecuador ha propuesto, al igual que se ha realizado en otros países, un diseño de Encuesta Sobre Las Condiciones De Seguridad Y Salud En El Trabajo (ECSST-EC) para Ecuador, que sirva de instrumento válido y fiable para la recolección y análisis de información.

\section{Materiales y Métodos}

La elaboración, construcción y diseño del cuestionario (ECSST-EC) se llevó a cabo en tres fases, a través de un proceso consensuado entre más de 40 expertos a nivel nacional e internacional.

En una primera fase, con el objeto de enmarcar el modelo en un contexto mundial que permita la comparabilidad de los datos obtenidos tanto a nivel regional como internacional, se procedió con la revisión documental, análisis estructural y de diseño de más de 20 encuestas internacionales y estudios referentes a este tema.

Entre los principales organismos que han realizado aportes en este tema, se encuentra la Fundación Europea para la Mejora de las Condiciones de Vida y de Trabajo (Eurofound), que tiene como función aportar conocimientos sobre las condiciones de vida, trabajo y relaciones industriales para la formulación de políticas sociales y laborales ${ }^{21}$.

Eurofound ha realizado una relevante contribución en este ámbito a través de la Encuesta Europea sobre las Condiciones de Trabajo (EWCS por sus siglas en Ingles); estas forman parte de los sistemas de información en salud laboral y tienen como objetivo específico aportar información sobre la percepción de los trabajadores respecto a la exposición a factores de riesgo laboral para la toma de decisiones entre los países de la Unión Euporea (EU); en la última encuesta se entrevistaron a domicilio 43.000 trabajadores de 35 países pertenecientes a la EU.

En la presente fase de estudio se procedió a la revisión de las seis EWCS que hasta el momento Eurofound ha aplicado $22 ; 25$.

En Estados Unidos el Instituto Nacional para la Salud y Seguridad Ocupacional (National Institute of Occupational Safety and Health - NIOSH), como organismo encargado de realizar investigaciones y recomendaciones para la prevención de enfermedades y lesiones relacionadas con las condiciones de trabajo, ha realizado dos encuestas ${ }^{26 ; 28}$.

España, como país pionero en la aplicación de encuestas sobre condiciones de trabajo, a través del Instituto Nacional de Seguridad e Higiene en el Trabajo (INSHT), ha realizado periódicamente siete Encuestas Nacionales sobre Condiciones de Trabajo (ECTS) desde $1987^{18}$. 
En lo referente a Latinoamérica se han realizado siete encuestas desde el 2007, Tabla 1.

\section{Tabla 1. Encuestas sobre Condiciones de Seguridad y Salud en el Trabajo realizadas en Centro y Latinoamérica, 2007-2013.}

\begin{tabular}{|c|c|c|c|}
\hline AÑO & REF. & DENOMINACIÓN & PAÍS \\
\hline 2007 & I ENCST & $\begin{array}{l}\text { Primera Encuesta Nacional de Condiciones de } \\
\text { Salud y Trabajo en el Sistema General de Riesgos } \\
\text { Profesionales }\end{array}$ & Colombia \\
\hline 2007 & ENCTSS & $\begin{array}{l}\text { Encuesta Nacional sobre Condiciones de } \\
\text { Trabajo, Salud y Seguridad Ocupacional }\end{array}$ & Guatemala \\
\hline 2009 & ENT & $\begin{array}{l}\text { Encuesta Nacional a Trabajadores, Empleo, } \\
\text { Trabajo, Condiciones y Medio Ambiente Laboral }\end{array}$ & Argentina \\
\hline 2009-2010 & I ENETS & $\begin{array}{l}\text { Primera Encuesta Nacional de Empleo, Trabajo, } \\
\text { Salud y Calidad de Vida de los Trabajadores y } \\
\text { Trabajadoras en Chile }\end{array}$ & Chile \\
\hline 2011 & IECCTS & $\begin{array}{l}\text { Encuesta Centroamericana sobre Condiciones de } \\
\text { Trabajo y Salud }\end{array}$ & $\begin{array}{l}\text { Costa Rica, El Salvador, } \\
\text { Guatemala, Honduras, } \\
\text { Nicaragua y Panamá }\end{array}$ \\
\hline 2012 & ECTSS & $\begin{array}{l}\text { Encuesta sobre Condiciones de Trabajo, } \\
\text { Seguridad y Salud Laboral en Uruguay }\end{array}$ & Uruguay \\
\hline 2013 & II ENCST & $\begin{array}{l}\text { Segunda Encuesta Nacional de Condiciones de } \\
\text { Salud y Trabajo en el Sistema General de Riesgos } \\
\text { Profesionales }\end{array}$ & Colombia \\
\hline
\end{tabular}

A nivel nacional la información que se puede resaltar de utilidad para el presente estudio, son las recabadas por las encuestas desarrolladas por el Instituto Nacional de Estadística y Censos (INEC) del Ecuador, relacionadas con las condiciones de empleo, salud y calidad de vida de la población ecuatoriana, las mismas fueron revisadas en esta primera fase, de igual modo, cabe mencionar la aplicación de las Encuestas de Condiciones de Vida (ECV) que se han aplicado desde 1994, permitiendo obtener indicadores sobre los niveles de vida y el bienestar de la población relacionando factores como educación, salud, pobreza e inequidad para la aplicación de política.

Siguiendo con el proceso de revisión documental, se analizaron otros estudios relacionados ${ }^{29 ; 55}$, que permitieron conocer la temática objeto de estudio y dar sustento para la confección del cuestionario. 
El grupo de investigadores y colaboradores revisó y analizó la información de manera objetiva y crítica. En relación a esta propuso aspectos de intervención y discusión que sirvieron de base para la fase II.

La segunda fase tuvo como propósito la confección del cuestionario, esta se desarrolló en dos partes, la primera de ellas con el objetivo de determinar las dimensiones y sub-dimensiones y la segunda para establecer y elaborar las variables e Ítems que conformarían el cuestionario.

Tras la revisión documental, los investigadores y colaboradores participaron en dos talleres presenciales que buscaron consensuar las dimensiones y sub-dimensiones a incluir en el cuestionario, la propuesta de las mismas se sustentó a su vez en el marco teórico de la seguridad y salud ocupacional y la revisión analítica vía correo electrónico de los resultados previos consensuados. El primer resultado arrojó 9 dimensiones y 26 sub dimensiones, Tabla 2.

Durante los meses de diciembre 2015 y enero 2016, se realizó un análisis exhaustivo de las dimensiones y sub-dimensiones propuestas y en un segundo consenso el grupo de expertos e investigadores sustentan la decisión de pasar de 9 a 7 dimensiones y de 26 a 23 sub-dimensiones.

Continuando el proceso de construcción planteado, en la segunda etapa de esta fase, se determinaron las variables de cada sub-dimensión, así como, la redacción más adecuada para cada pregunta. Para la confección de cada una de las preguntas del cuestionario, se elaboró una plantilla, con el objeto de redactar y formular la pregunta en función al objetivo de cada variable, sustentado en el marco teórico de las dimensiones y sub dimensiones. Como resultado se obtuvo un primer borrador conformado por 77 preguntas.

Una vez confeccionado este primer borrador, se prosiguió con la tercera fase de la investigación, que consistió en la revisión y retroalimentación del cuestionario por parte de investigadores y colaboradores externos, así como la estimación de la fiabilidad de la consistencia interna del cuestionario por medio de la aplicación del Alfa de Cronbach.

Tabla 2. Listado Preliminar de dimensiones y sub-dimensiones.

\begin{tabular}{cc}
\hline Dimensiones & Sub Dimensiones \\
\hline Sociodemográficas Y Laborales & Demograficas \\
Caracteristicas Familiares & Laborales \\
& Estructura Familiar \\
Condiciones De Empleo & Cuidado De Personas \\
& Trabajo Doméstico \\
& Protección Social \\
Condiciones De Trabajo: Higiene Industrial & Relación Laboral \\
\hline
\end{tabular}




\begin{tabular}{cc}
\hline & Químicas \\
Condiciones De Trabajo: Ergonómicas & Biológicas \\
Condiciones De Trabajo: Seguridad Industrial & Ergonómicas \\
Condiciones De Trabajo: Psicosociología & Seguridad \\
& Mecanicos \\
Salud Ocupacional & Control \\
& Demandas \\
Recursos Y Actividades Preventivas & Recompensa \\
& Salud Autopercibida \\
& Salud Mental \\
& Accidentes De Trabajo \\
& Enfermedades Profesionales \\
& Incapacidades \\
& Actividades \\
& Recursos \\
\hline
\end{tabular}

En esta fase el cuestionario fue enviado vía correo electrónico al grupo de colaboradores externos, equipo internacional multidisciplinar de expertos pertenecientes a instituciones universitarias y empresas privadas del sector relacionado con la medicina del trabajo, Tabla 3 ., para su revisión, aporte y retroalimentación. Fruto de este análisis se recibió informes detallados por parte de cada equipo.

\section{Tabla 3. Relación de revisores externos por ciudad}

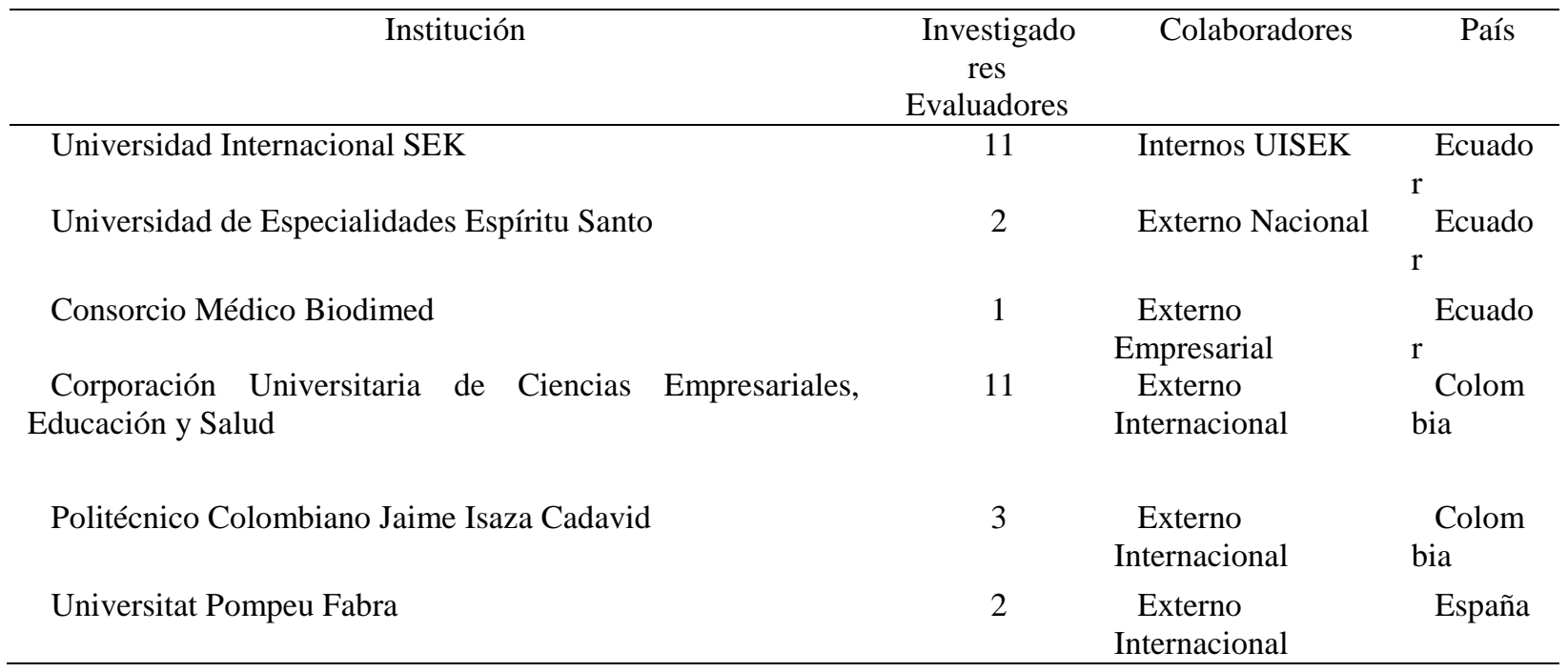

La información recibida fue estudiada por el grupo de investigadores y se usó para la reformulación del cuestionario y la elaboración de un segundo borrador que pasó de 77 a 79 
preguntas y de 23 a 26 subdimenciones. Se incluyeron dos preguntas recomendadas por el Centro de Investigación en Salud Laboral (CISAL) de la Universitat Pompeu Fabra y Red de Expertos en Encuestas de Condiciones de Trabajo, Empleo y Salud (RED ECTS), P2. ¿Podría decirme su edad? en años y P9.1 ¿Cuántas horas trabaja a la semana en su trabajo principal?.

A fin de dar validez y evaluar la consistencia interna del cuestionario, se realizó una prueba piloto a 26 encuestados (hombres y mujeres) de los sectores norte, centro y sur de la ciudad de Quito, en el período del 25 al 29 de abril del 2016.

Si bien, los resultados de la prueba piloto fueron satisfactorios, indicaron la necesidad de ajustar el lenguaje en la formulación de algunas preguntas para mejorar la comprensión y la aclaración a los encuestadores para identificar con claridad la profesión, actividad económica y molestias o problemas de salud.

\section{Resultados}

Como producto de la investigación se obtuvo un cuestionario final (Anexo 1.) que contiene 7 dimensiones, 26 sub-dimensiones y un total de 79 preguntas.

La dimensión sociodemográfica y laboral se ha dividido en 3 sub-dimensiones; demográfica (4), social (1) y laboral (4), conformando un total de 9 preguntas en el cuestionario.

Para la dimensión condiciones de empleo se ha considerado obtener información sobre el tiempo de trabajo (sub-dimensión), estableciéndose preguntas sobre el tiempo de trabajo en la empresa (2), tipo de jornada laboral (1) y turno de trabajo (1) conformando un total de 4 preguntas en el cuestionario.

La dimensión de seguridad en el trabajo se ha dividido en 2 sub-dimensiones; exposición a riesgos mecánicos (15) y equipos de protección personal (2), conformando un total de 17 preguntas en el cuestionario.

La dimensión higiene industrial engloba 3 sub-dimensiones y sus respectivas preguntas de la encuesta; agentes físicos (8), agentes químicos (4) y agentes biológicos (1), conformando un total de 13 preguntas en el cuestionario.

La dimensión ergonomía engloba 4 sub-dimensiones con sus respectivas preguntas; posición de trabajo (1), posturas forzadas (1), manipulación manual de cargas (1) y movimientos repetitivos (1), conformando un total de 4 preguntas en el cuestionario.

La dimensión psicosocial se ha clasificado en dos grupos; factores intra-laborales y extralaborales. Para los factores intra-laborales se ha divido en 4 sub-dimensiones; control (2), demanda psicológica (3), apoyo social (2) e inseguridad en el empleo (1) y, extra-laboral en 2 subdimensiones; estilo de vida (4) y conciliación con la vida laboral y socio-familiar (2), conformando un total de 14 preguntas en el cuestionario.

La dimensión salud ocupacional o estado de salud del trabajador engloba 5 sub- 
dimensiones; salud auto-percibida (12), salud mental (1), accidentes de trabajo (2) y enfermedades profesionales (1), en éstas dos últimas sub-dimensiones, se consideran las incapacidades (2) derivadas por accidente y/o enfermedad, conformando un total de 18 preguntas en el cuestionario.

Tabla 4. Estructura de la Encuesta de condiciones de Seguridad y Salud en el Trabajo (I ECSSO-EC)

\begin{tabular}{|c|c|c|c|c|}
\hline Dimensión & Sub-Dimensión & $\begin{array}{c}\mathrm{N}^{\circ} \\
\text { Preguntas }\end{array}$ & Total & $\begin{array}{c}\% \\
\text { Cuestionario } \\
\end{array}$ \\
\hline \multirow[t]{3}{*}{ Sociodemográfica Y Laboral } & Demográfica & 4 & 9 & $11,4 \%$ \\
\hline & Social & 1 & & \\
\hline & Laboral & 4 & & \\
\hline \multirow[t]{3}{*}{ Condiciones De Empleo } & Tiempo De Trabajo & 2 & 4 & $5,1 \%$ \\
\hline & Jornada Laboral & 1 & & \\
\hline & Turno De Trabajo & 1 & & \\
\hline \multirow[t]{2}{*}{ Seguridad En El Trabajo } & Exposición A Riesgos Mecánicos & 15 & 17 & $21,5 \%$ \\
\hline & Equipos De Protección Personal & 2 & & \\
\hline \multirow[t]{3}{*}{ Higiene Industrial } & Agentes Físicos & 8 & 13 & $16,5 \%$ \\
\hline & Agentes Químicos & 4 & & \\
\hline & Agentes Biológicos & 1 & & \\
\hline \multirow[t]{4}{*}{ Ergonomía } & Posición De Trabajo & 1 & 4 & $5,1 \%$ \\
\hline & Posturas Forzadas & 1 & & \\
\hline & Manipulación Manual De Cargas & 1 & & \\
\hline & Movimientos Repetitivos & 1 & & \\
\hline \multirow[t]{6}{*}{ Psicosociología } & Control & 2 & 14 & $17,7 \%$ \\
\hline & Demanda Psicológica & 3 & & \\
\hline & Apoyo Social & 2 & & \\
\hline & Inseguridad Empleo & 1 & & \\
\hline & Estilo De Vida & 4 & & \\
\hline & Conciliación & 2 & & \\
\hline \multirow[t]{5}{*}{ Salud Ocupacional } & Salud Auto-Percibida & 12 & 18 & $22,8 \%$ \\
\hline & Salud Mental & 1 & & \\
\hline & Accidentes De Trabajo & 2 & & \\
\hline & Enfermedades Profesionales & 1 & & \\
\hline & Incapacidades & 2 & & \\
\hline
\end{tabular}


Los resultados de la prueba piloto sirvieron como base para estimar estadísticamente la fiabilidad de la consistencia interna de la encuesta; estos se evaluaron a través del Alfa de Cronbach, obteniendo como resultado 0.856 .

La encuesta diseñada permitirá ser en futuro una herramienta, válida y fiable para la recolección de datos en Ecuador, su aplicación permitirá identificar los factores de riesgo laboral presentes en los lugares de trabajo y su impacto en la salud de la población trabajadora, así como, su comparación con los resultados de otras encuestas a nivel internacional. 


\section{Bibliografía}

Consejo Nacional de Seguridad y Salud Ocupacional. (2007). Encuesta Nacional sobre Condiciones de Trabajo, Salud y Seguridad Ocupacional Guatemala. [consultado 23 de noviembre de 2015]. Disponible en: http://unpan1.un.org/intradoc/groups/public/documents/icap/unpan032002.pdf

Ministerio de Protección Social. (2007). Primera Encuesta Nacional de Condiciones de Salud y Trabajo en el Sistema General de Riesgos Profesionales (I ENCST). [consultado 26 de noviembre de 2015]. Disponible en: http://www.oiss.org/estrategia/IMG/pdf/I_encuesta_nacional_colombia2.pdf

Ministerio del Trabajo. (2013). Informe Ejecutivo. Segunda Encuesta Nacional de Condiciones de Salud y Trabajo en el Sistema General de Riesgos Profesionales (II ENCST). [consultado 28 de noviembre de 2015]. Disponible en: http://ccs.org.co/salaprensa/images/Documentos/Informe_Ejecutivo_Ii\%20encsst.pdf

Ministerio de Trabajo, Empleo y Seguridad Social, Superintendencia de Riesgos del Trabajo. (2009). Encuesta Nacional a Trabajadores, Empleo, Trabajo, Condiciones y Medio Ambiente Laboral. [consultado 28 de noviembre de 2015]. Disponible en:

http://www.srt.gob.ar/images/Publicaciones/Informes_investigacion/Libro_Final_Corregido_201 4.pdf

Ministerio de Salud, Dirección del Trabajo, Instituto de Seguridad Laboral. (2009-2010). Primera Encuesta Nacional de Empleo, Trabajo, Salud y Calidad de Vida de los Trabajadores y las Trabajadoras en Chile. [consultado 28 de noviembre de 2015]. Disponible en: http://www.superacionpobreza.cl/wpcontent/uploads/2014/03/primera_encuesta_nacional_empleo.pdf

OISS. (2012). I Encuesta sobre Condiciones de Trabajo, Seguridad y Salud Laboral en Uruguay. [consultado 30 de noviembre de 2015]. Disponible en: http://www.oiss.org/estrategia/IMG/pdf/Encuesta_Uruguay.pdf.

OISS, INSHT. (2011). Encuesta Centroamericana Sobre Condiciones de Trabajo y Salud. [consultado 30 de noviembre de 2015]. Disponible en: http://www.oiss.org/estrategia/encuestas

Instituto Nacional de Seguridad e higiene en el trabajo INSHT. (2003): V Encuesta Nacional de Codiciones de Trabajo [ consultado 26 de noviembre 2015]. Disponible en:

http://www.insht.es/Observatorio/Contenidos/InformesPropios/Desarrollados/Ficheros/Informe_ V_ENCTDic2010.pdf

Instituto Nacional de Seguridad e higiene en el trabajo INSHT. (2011): VII Encuesta Nacional de Codiciones de Trabajo [ consultado 26 de noviembre 2015]. Disponible en: http://www.insht.es/InshtWeb/Contenidos/Documentacion/Fichas\%20de\%20publicacion es/En\%20catalogo/Observatorio/Informe\%20(VII\%20ENCT).pdf 
Instituto de Seguridad y Salud Laboral. I Encuesta Regional de Condiciones de Trabajo. Comunidad Autónoma de la Región de Murcia; 2005. [consultado 3 de febrero de 2016]. Disponible

en: https://www.carm.es/web/pagina?Idcontenido=3637\&Idtipo=100\&Rastro=C721\$M3132 1

Instituto de Seguridad y Salud Laboral. II Encuesta Regional de Condiciones de Trabajo. Comunidad Autónoma de la Región de Murcia; 2010. [consultado 3 de febrero de 2016]. Disponible en:https://www.carm.es/web/pagina?Idcontenido=29900\&IDTIPO=100\&RASTRO=c72 $1 \$ \mathrm{~m} 31321)$

(Instituto de Seguridad y Salud Laboral. II Encuesta Regional de Condiciones de Trabajo en Empresas. Comunidad Autónoma de la Región de Murcia; 2011. [consultado 3 de febrero de 2016]. Disponible en: https://Www.Carm.Es/Web/Pagina?Idcontenido=39225\&IDTIPO=100\&RASTRO=c721 $\$ \mathrm{~m} 31321)$

Itati Iñiguez MJ, Agudelo Suárez AA, Campos Serna J, Cornelio CI y Benavides F.G. Encuestas de condiciones de trabajo y salud: su utilización en la investigación en salud laboral. Medicina y Seguridad del Trabajo. 2012; 58 (228); 205-215.

Lucchini RG and London L. Global occupational health: current challenges and the need for urgent action. Ann Glob Health. 2014; 80(4): 251-256.

Eurofound. (2007). Working Conditions Survey-A comparative analysis (2005/2016). [citado 11 noviembre 2015]. Disponible en: http://www.eurofound.europa.eu/publications/htmlfiles/ef0744.htm.

Narocki C, Zimmerman M, Artazcoz L, Gimeno D y Benavides FG. Encuestas de condiciones de trabajo y salud en España: comparación de los contenidos del cuestionario del trabajador. Arch Prev Riesgos Labor. 2009; 12 (2): 14-8.

VIOSH. Australia and the University of Vallarta. International Review of Surveillance and control of Workplaces exposures. Wellington: NOHSAC Technical Report 5, 2006.

Claudia Narocki, M. et al. (2007). Estudio comparado de las Encuestas sobre Condiciones de Trabajo y Salud realizadas en España. Observatorio de Salud Laboral.

Metodología de la Encuesta de Condiciones de Vida ECV, 2013- 2014. Instituto Nacional de Estadística y Censos. [consultado 16 de diciembre de 2015]. Disponible en: http://www.ecuadorencifras.gob.ec/documentos/webinec/ECV/ECV_2015/documentos/Metodologia/Documento\%20Metodologico\%20ECV $\% 206 R . p d f$. 
Freire, W.B., et al. (2014). Tomo I: Encuesta Nacional de Salud y Nutrición de la población ecuatoriana de 0 a 59 años. ENSANUT-ECU 2012. Ministerio de Salud Pública. Instituto Nacional de Estadística y Censos. [consultado 16 de diciembre de 2015]. Disponible en: http://www.ecuadorencifras.gob.ec/documentos/webinec/Estadisticas_Sociales/Ensanut/Msp_Ensanut-ECU_06-10-2014.pdf.

Wegman, D. and Hogstedt, C. (2014). If it's not counted it didn't happen!. Occup Environ Med; 71: pp. 457-458.

Primeros resultados: Sexta Encuesta europea sobre las condiciones de trabajo. [consultado 6 de febrero de 2016]. Disponible en: https://www.eurofound.europa.eu/sites/default/files/ef_publication/field_ef_document/ef 1568es.pdf

(Encuestas. [consultado 3 de febrero de 2016]. Disponible en: https://www.eurofound.europa.eu/es/surveys

Parent-Thirion, A., et al. (2012). Fifth European Working Conditions Survey - Overview report. Luxembourg: Eurofound, Publications Office of the European Union.

Cabrita, J. y Peycheva, D. (2014). National working conditions surveys in Europe: A compilation. Luxembourg: Eurofound, Publications Office of the European Union.

NIOSH. National occupational exposure survey field guidelines. Vol. I. Publication No. 88-106, 1988. [consultado 20 diciembre de 2015]. Disponible de: http://www.cdc.gov/niosh/88106.html.

NIOSH. Prevención de exposiciones de paramédicos a agentes patógenos transmitidos por la sangre. DHHS (NIOSH) Publicación N. ${ }^{\circ}$ 2010-139. [consultado 29 diciembre de 2015]. Disponible en: http://www.cdc.gov/spanish/niosh/docs/wp-solutions/2010-139_sp.

McAdams, M.T., Kerwin, J.J., Olivo, V. and Goksel, H.A. (2011). National Assessment of the Occupational Safety and Health Workforce. National Institute for Occupational Safety and Health. [consultado 21 diciembre de 2015]. Disponible en: http://www.cdc.gov/niosh/oshworkforce/pdfs/NASHW_Final_Report.pdf.

Benach, J. y Benavides, F.G. (2013). Precarious employment and health-related outcomes in the European Union. Luxembourg: Eurofound, Publications Office of the European Union.

Agostini, M., et al. (2014). Industrial relations practices related to psychosocial constraints at work in the steel sector. Luxembourg: Eurofound, Publications Office of the European Union.

Aumayr Pintar, C. et al. (2015). Developments in working life in Europe: EurWORK annual review 2014. Luxembourg: Eurofound, Publications Office of the European Union.

Resolución 957. (2005). Reglamento al Instrumento Andino de Seguridad y Salud en el Trabajo. 
Comunidad Andina de Naciones.

Instituto Salud y Trabajo (ISAT). (2011). Diagnóstico Situacional en Seguridad y Salud en el Trabajo. [consultado 11 de febrero de 2016]. Disponible en: http://www.comunidadandina.org/camtandinos/OLA/Documentos/Pdf/Diagnostico-SSTEcuador_ISAT_2011.pdf

Instituto Salud y Trabajo (ISAT). (2010). Percepciones sobre condiciones de Seguridad y Salud en el Trabajo en la Región Andina. [consultado 11 de febrero de 2016]. Disponible en: http://intranet.oit.org.pe/WDMS/bib/virtual/coleccion_tem/seg_trab/percepciones_condit _sst[region_andina].pdf

Instituto de Salud y Trabajo (ISAT). (2011). Diagnóstico situacional en seguridad y salud en el trabajo - Ecuador. Lima.

OISS. (2012). Indicadores de siniestralidad laboral en Iberoamérica. [consultado 17 de febrero de 2016].

Disponible

en http://www.oiss.org/estrategia/IMG/pdf/Informe_sobre_siniestralidad.pdf

OIT. Principales estadísticas (anuales). [consultado 18 de febrero de 2016]. Disponible en: http://laborsta.ilo.org/STP/guest

Japan Industrial Safety \& Association, JISHA. (2010). Statistics of Workers' Health Condition in Japan. Tokyo: JISHA.

Cabinet Office, Government of Japan. (2012). White paper on Suicide Prevention in Japan. Tokyo: Cabinet Office.

Choi, E. and Ha, Y. (2009). Work-related Stress and Risk Factors among Korean Employees in Journal of the Korean Academy of Nursing; Vol. 39 (4): pp. 549-561

Kim, Y.S. et al. (2015). A Comparison between the Second Korean Working Conditions Survey (KWCS) and the First KWCS in Safety and Health at Work; Vol. 6 (2): pp. 85-89.

Resumen Ejecutivo. VII Encuesta Nacional de Condiciones de Trabajo. INSHT. [consultado 17 de febrero de 2016]. Disponible en: http://www.insht.es/InshtWeb/Contenidos/Documentacion/Fichas\%20de\%20publicacion es/En\%20catalogo/Observatorio/Informe\%20(Vii\%20enct).pdf.)

Daubas-Letourneux, V. y Thébaud-Mony, Annie (2012). Work organization and health at work in the European Union. Luxembourg: Eurofound, Publications Office of the European Union.

Ardito, C., et al. (2013). Health and well-being at work. Luxembourg: Eurofound, Publications Office of the European Union.

Benach, J. y Benavides, F.G. (2013). Precarious employment and health-related outcomes in the 
European Union. Luxembourg: Eurofound, Publications Office of the European Union.

Agostini, M., et al. (2014). Industrial relations practices related to psychosocial constraints at work in the steel sector. Luxembourg: Eurofound, Publications Office of the European Union.

Aumayr-Pintar, C. et al. (2015). Developments in working life in Europe: EurWORK annual review 2014. Luxembourg: Eurofound, Publications Office of the European Union.

Ministère de l'emploi, du travail et de la cohésion sociale, Direction de l'Animation de la Recherche, des Études et des Statistiques (2005). Enquête sur 1'emploi 2005, questionnaire complémentaire sur les conditions de travail.

Nationale Enquête Arbeidsomstandigheden 2010. Nederlandse Organisatie voor Toegepast Natuurwetenschappelijk Onderzoek (TNO), Centraal Bureau voor de Sta- tistiek (CBS), Ministerie van Sociale Zaken en Werkgelegenheid. Nederland/The Netherlands.

NIOSH, OSHA. Current Intelligence Bulletin 28 - Vinyl Halides carcinogenicity. DHHS (NIOSH) Publication No. 79-102. [consultado 27 diciembre de 2015]. Disponible en: http://www.cdc.gov/niosh/79102_28.html.

Leiss, J.K., et al. (2006). Blood exposure among paramedics: incidence rates from the national study to prevent blood exposure in paramedics. Ann Epidemiol; 16 (9): pp. 720-725.

Leiss, J.K., Sousa, S. and Boal, W.L. (2009). Circumstances sur-rounding occupational blood exposure events in the na-tional study to prevent blood exposure in paramedics. In-dustrial Health; 47 (2): pp. 139-144.

Boal, W.L., et al. (2008). The national study to prevent blood exposure in paramed-ics: exposure reporting. Am J Ind Med; 51 (3): pp. 213-222.

Boal, W.L., et al. (2010). The national study to prevent blood ex-posure in paramedics: rates of exposure to blood. Int Arch Occup Environ Health; 83: pp. 191-199.

Mathews, R., et al (2008). Provision and use of personal protective equipment and safety devices in the national study to pre-vent blood exposure in paramedics. Am J Infect Control; 36 (10): pp. 743-749. 